\title{
Overcoming Microsoft Excel's weaknesses for crop model building and simulations
}

\begin{abstract}
Using spreadsheets such as Microsoft Excel for building crop models and running simulations can be beneficial. Excel is easy to use, powerful, and versatile, and it requires the least proficiency in computer programming compared to other programming platforms. Excel, however, has several weaknesses: it does not directly support loops for iterative calculations, and it does not allow one cell to alter the contents of another cell. Thus, the objective of this study was to develop an Excel add-in, called BuildIt, that overcomes some of Excel's weaknesses by: (1) providing a loop for repetitive calculations and (2) providing several operations (called actions) typically needed in building crop models. These actions are such as for numerical integration, initialization of variables, and solving differential equations using the Runge-Kutta method, as well as for copying and manipulation of cell ranges. BuildIt was written in Excel's script language, Visual Basic for Applications (VBA), but it does not require users to program in VBA to build their models. Several examples of models were used in this article to illustrate how BuildIt implements the infrastructure in Excel, and how it can be used to build models and run model simulations. With BuildIt, users are able to use Excel to build and run their mathematical models, without requiring any knowledge in VBA.
\end{abstract}

Keyword: Microsoft Excel; Building crop models; Weakness 